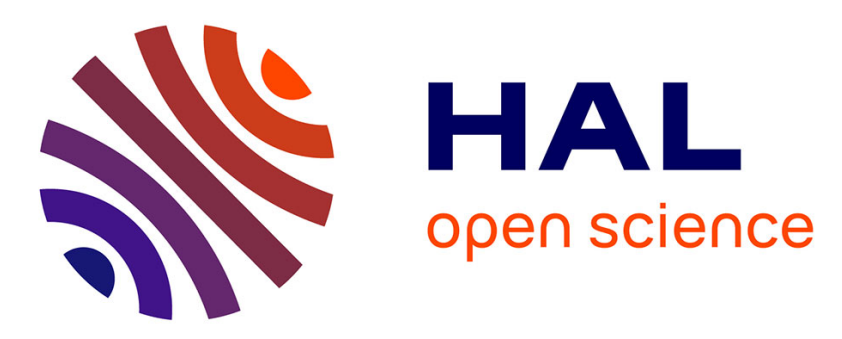

\title{
Estimating absolute aortic pressure using MRI and a one-dimensional model
}

\author{
Maya Khalifé, Astrid Decoene, Filipa Caetano, Ludovic de Rochefort, \\ Emmanuel Durand, Dima Rodríguez
}

\section{- To cite this version:}

Maya Khalifé, Astrid Decoene, Filipa Caetano, Ludovic de Rochefort, Emmanuel Durand, et al.. Estimating absolute aortic pressure using MRI and a one-dimensional model. Journal of Biomechanics, 2014, 47 (13), pp.3390-3399. hal-01083962

\section{HAL Id: hal-01083962 \\ https://hal.science/hal-01083962}

Submitted on 18 Nov 2014

HAL is a multi-disciplinary open access archive for the deposit and dissemination of scientific research documents, whether they are published or not. The documents may come from teaching and research institutions in France or abroad, or from public or private research centers.
L'archive ouverte pluridisciplinaire HAL, est destinée au dépôt et à la diffusion de documents scientifiques de niveau recherche, publiés ou non, émanant des établissements d'enseignement et de recherche français ou étrangers, des laboratoires publics ou privés. 


\section{$1 \quad$ Estimating absolute aortic pressure using MRI and a}

one-dimensional model

3 Maya Khalifé $^{\mathrm{a}}$, Astrid Decoene ${ }^{\mathrm{b}}$, Filipa Caetano ${ }^{\mathrm{b}}$, Ludovic de Rochefort $^{\mathrm{a}}$, $4 \quad$ Emmanuel Durand $^{\mathrm{a}}$, Dima Rodríguez ${ }^{\mathrm{a}}$

$5 \quad$ a Imagerie par Résonance Magnétique Médicale et Multi-Modalités (IR4M), Université

$6 \quad$ Paris-Sud-CNRS, UMR 8081, Orsay, France

$7 \quad{ }^{b}$ Laboratoire de Mathématiques d'Orsay, Université Paris-Sud - CNRS, UMR 8628, Orsay,

\section{Abstract}

Aortic blood pressure is a strong indicator to cardiovascular diseases and morbidity. Clinically, pressure measurements are done by inserting a catheter in the aorta. However, imaging techniques have been used to avoid the invasive procedure of catheterization. In this article, we combined MRI measurements to a one-dimensional model in order to simulate blood flow in an aortic segment. Absolute pressure was estimated in the aorta by using MRI measured flow as boundary conditions and MRI measured compliance as a pressure law for solving the model. Model computed pressure was compared to catheter measured pressure in an aortic phantom. Furthermore, aortic pressure was estimated in vivo in three healthy volunteers.

\section{Introduction}

To date, blood pressure (BP) is one of the most useful clinical indicator of 12 cardiovascular disease. Hypertension, more specifically, is a leading predictor of death in atherosclerosis diseases worldwide Cohn et al. [13], Collaboration

14 [14]. Therefore, measuring BP is of great interest for diagnosis and risk pre15 vention of cardiovascular events. An elevated pressure gives information about 16 the aortic state, the presence of atherosclerotic plaques, stenosis, calcification 
17 or aneurisms. In a clinical routine, a sphygmomanometer is used to measure

18 systolic and diastolic brachial pressure. However, due to reflexion in the distal

19 arteries, the aortic pressure waveform is altered while traveling through the vas-

20 cular system. Thus distortion of the wave shape as well as systolic amplification

21 occur on the systolic pressure measured in the brachial artery O'rourke et al.

22 [32], Park and Guntheroth [34], Salvi [37]. Although models and transfer func-

23 tions to link brachial BP to aortic pressure exist Chen et al. [12, 11], Liang [26],

24 wave reflection in the arterial system makes it difficult to reproduce the wave

25 contour with great fidelity from such methods. Until now, the gold-standard of

26 aortic pressure measurement is catheterization, which is invasive and not repeat-

${ }_{27}$ able in a routine procedure Murgo et al. [30], Skinner and Adams [40]. In recent

28 years, imaging techniques have been used to assess pressure gradients from ve-

29 locity or acceleration maps and its combination with fluid mechanics equations

sо has been exploited in order to measure the BP non-invasively. Doppler Ultra-

31 sound (US) used to measure blood velocity in the arteries was associated to the

32 standard simplified Bernoulli equation in order to determine pressure differences

${ }_{33}$ between two measurement sites. This latter technique is reported to be user-

34 dependent and error-prone due to the wave angle of incidence Zananiri et al.

35 [51], Muhler et al. [29], hence its accuracy in determining the maximum velocity

36 in the artery is debatable. Also, extending US to other situations is limited

37 by the inapplicability of Bernoulli equation to unsteady flows Yang et al. [50].

38 Phase-Contrast (PC) MRI allows accurate encoding of the blood velocity in the

39 arteries in the three directions; hence, it has been largely used for non-invasive

40 pressure estimation. Some authors computed pressure differences using Poisson

41 equation Yang et al. [50], others integrated the Navier-Stokes (NS) equations

42 using MRI velocity maps Tyszka et al. [45], Thompson and McVeigh [44], Bock

43 et al. [8], Ebbers et al. [17] or acceleration maps to avoid computational errors 
44 arising from velocity derivation Buyens et al. [10]. These methods compute a

45 pressure gradient, and to estimate an absolute pressure, require a zero-pressure

46 reference point which has to be measured with a catheter, or user-defined in

47 a gross assumption. Consequently, these methods are not an alternative to

48 catheter measurements, which remain more accurate. In this work, we propose

49 a non-invasive technique to extract absolute pressure in a straight artery from

so MR velocimetry using a biomechanical one-dimensional (1D) model as proposed

51 by Formaggia et al. Formaggia et al. [18]. Although a three-dimensional (3D)

52 model gives a more complete and realistic reproduction of the aortic flow, 1D

53 models are able to describe the non-linear flow behavior in larger elastic vessels

54 Hughes and Lubliner [22]. As these models are reasonably accurate, they are

55 widely used for aortic flow simulations. Their accuracy has been assessed by

56 comparison with experimental data acquired in a tube phantom Bessems et al.

57 [7], in a distributed arterial model Olufsen et al. [31], Alastruey et al. [2] and in

58 vivo Reymond et al. [36], Alastruey et al. [3]. Furthermore, the 1D model relies

59 on the establishment of a pressure law consisting of a relation between pressure

60 and vessel section area. The pressure laws used in 1D model equations are deter-

${ }_{61}$ mined experimentally with invasive measurements or estimated using complex

62 algorithms. Additionally, these pressure laws are complicated and involve the

${ }_{63}$ determination of multiple parameters. Here, we propose a pressure law based

64 on the aortic compliance which reflects arterial elasticity and can be determined

65 non-invasively with MRI. Using this pressure law, we coupled the 1D model with

66 realistic boundary conditions measured by MRI to estimate absolute pressure

67 in the aortic segment. The derived model was tested on a straight compliant

68 phantom and computed pressure was compared to experimental pressure mea-

69 surements recorded simultaneously with the MRI acquisition. The model was

7o also tested on a real-sized compliant aortic phantom Then, the model is used to 
82 For large arteries such as the aorta, it is a safe assumption to consider a flat 83 velocity profile for the boundary layer is very thin compared to the vessel radius

84 Olufsen et al. [31].

85 The main variables of the problem are (Figure 1):

86

- axial section area $A$

$$
A(t, z)=\int_{S(t, z)} \mathrm{d} \sigma
$$

87

- mean flow $Q$

$$
Q(t, z)=\int_{S} u_{z} \mathrm{~d} \sigma
$$

s8 $\quad$ blood pressure $P(t, z)$,

89 where $\mathrm{d} \sigma$ denotes the area element. Their evolution is described by the mo- 
constant viscosity:

$$
\begin{aligned}
\frac{\partial A}{\partial t}+\frac{\partial Q}{\partial z} & =0 \\
\frac{\partial Q}{\partial t}+\frac{\partial}{\partial z}\left(\frac{Q^{2}}{A}\right)+\frac{A}{\rho} \frac{\partial P}{\partial z}+K_{r}\left(\frac{Q}{A}\right) & =0
\end{aligned}
$$

$92 K_{r}$ is the friction coefficient; for a flat profile in blood flow problems, $K_{r}=22 \pi \nu$,

93 where $\nu$ is the vessel wall kinematic viscosityFormaggia and Veneziani [19]. $\rho$ is

94 the blood density.

$95 \quad$ Pressure law

96 To close the system, a relation between the section area $A$ and the pressure

97 $\quad P$ is defined. This pressure law depends on section area $A_{0}(z)=\pi R_{0}^{2}(z)$ at time 98 $t=0$ and on a set of parameters $\beta=\left(\beta_{0}, \beta_{1}, \ldots \beta_{n}\right)$ related to the vessel wall

99 physical and mechanical properties. $P_{\text {ext }}$ is the external pressure exerted by the

100

101

follows Quarteroni and Formaggia [35], Alastruey [1]:

$$
P-P_{\text {ext }}=\beta_{0} \frac{\sqrt{A}-\sqrt{A_{0}}}{A_{0}}
$$

${ }_{107} \beta_{0}=\frac{\sqrt{\pi} h_{0} E}{1-\xi^{2}}$ using Young's modulus $E$ and the vessel thickness $h_{0}$ and Poisson's

A more general law proposed in Hayashi et al. [21], Smith and Hunter [41] 110 is written as: 


$$
P-P_{e x t}=\beta_{0}\left[\left(\frac{A}{A_{0}}\right)^{\beta_{1}}-1\right] .
$$

The parameters $\beta_{0}$ and $\beta_{1}$ values can be obtained either by fitting experimental pressure versus section measurements Smith [42], or by solving an inverse problem with a 3D-model solution Martin et al. [27], Dumas [16]. Hence, these laws cannot be determined non-invasively, and need knowledge about the vessel properties. Additionally, they seem too complex to determine during a clinical application. Finding a simple non-invasive way to determine a pressure law is of great interest, consequently, we turned to the compliance. Indeed, in physiological conditions, the aorta section deformation is commonly assumed to be linked to the intravascular pressure by the aortic compliance Langewouters et al. [24]. Aortic compliance establishes a linear relation between the pressure and section area; it represents the arterial wall ability to deform in response to a pressure variation Conrad [15]. Also, it is clinically used and can be estimated non-invasively by measuring the pulse wave velocity (PWV) in MRI. Hence, it provides a simple and non-invasive pressure law that can be applied in vivo and, as it includes compliance changes, is patient-specific.

In fact, compliance is given by the ratio of section variation to pressure variation :

$$
\mathcal{C}=\frac{d A}{d P_{t}}
$$

where $P_{t}=P-P_{e x t}$ is the transmural pressure. The compliance is considered as a local constant on an arterial segment. By integrating equation (7) and knowing that when $P_{t}=0$, i.e. $P=P_{e x t}, A=A_{0}$, we write $A=\mathcal{C} P_{t}+A_{0}$ where $A_{0}$ is the section area at the equilibrium state. 
132 We write equation 7 as $\mathcal{C}=\frac{A-A_{0}}{P-P_{\text {ext }}}$, thus deriving the pressure law:

$$
P-P_{\text {ext }}=\frac{A_{0}}{\mathcal{C}}\left(\frac{A}{A_{0}}-1\right)
$$

133 In the limit of small displacements, this pressure law is equivalent to the com-

134 monly used linear law $((5))$ for

$$
\beta_{0}=\frac{2 A_{0}^{3 / 2}}{\mathcal{C}}
$$

135 In fact, by linearizing $((5))$ (see also Alastruey et al. [5]), we have:

$$
\beta_{0} \frac{\sqrt{A}-\sqrt{A_{0}}}{A_{0}}=\frac{\beta_{0}}{2 A_{0}^{3 / 2}}\left(A-A_{0}\right)+O\left(A-A_{0}\right)^{2}
$$

136

137

138

$\bullet \mathbf{U}=\left[\begin{array}{l}A \\ Q\end{array}\right]$

- $\mathbf{F}=\left[\begin{array}{c}Q \\ \frac{Q}{A}^{2}+C_{1}\end{array}\right], C_{1}(A)=\int_{0}^{A} c_{1}^{2}(\tau) d \tau$ and $c_{1}=c_{1}(A)=\sqrt{\frac{A}{\rho} \frac{\partial P}{\partial A}}$,

$\bullet \mathbf{S}=\left[\begin{array}{c}0 \\ -K_{r} \frac{Q}{A}\end{array}\right]$

142 Since $A>0$, the matrix $\mathbf{D F}$ possesses two real distinct eigenvalues $\lambda_{ \pm}=\frac{Q}{A} \pm c_{1}$

143 and system (10) is a hyperbolic system of partial differential equations For-

144 maggia and Veneziani [19]. The blood flow is assumed to be sub-critical, thus

$145 \lambda_{-}<0$, and $\lambda_{ \pm}$have opposite signs. 

153 diagonal variables $\left(W_{1}, W_{2}\right)$ as

$$
A=\left(\sqrt{\rho \mathcal{C}} \frac{W_{1}-W_{2}}{4}\right)^{2}, \quad Q=A \frac{W_{1}+W_{2}}{2} .
$$

154 For system (10) approximation, a finite volume scheme is defined by a grid over 156 given by $\left[z_{\mathfrak{i}}, z_{i+1}\left[\times\left[t^{n}, t^{n+1}\left[\right.\right.\right.\right.$, where $z_{\mathfrak{i}}=\mathfrak{i} \Delta z, \mathfrak{i}=0, \ldots, M$, with $M \Delta z=L$, 157 and $t^{n}=n \Delta t^{n}, n=0, \ldots, N$, with $N \Delta t^{N}=T$. We seek $U_{i}^{n}$ approximating $158 \quad \frac{1}{\Delta z} \int_{z_{\mathrm{i}}}^{z_{\mathrm{i}+1}} U\left(x, t^{n}\right) \mathrm{d} z$.

159 The numerical scheme is a classical finite volume scheme of Rusanov type 160

$$
W_{1}=\frac{Q}{A}+2 \sqrt{\frac{A}{\rho \mathcal{C}}}, \quad W_{2}=\frac{Q}{A}-2 \sqrt{\frac{A}{\rho \mathcal{C}}} .
$$

where $W=\left(W_{1}, W_{2}\right)$ are the characteristic variables, $\boldsymbol{\Lambda}=\left[\begin{array}{cc}\lambda_{+} & 0 \\ 0 & \lambda_{-}\end{array}\right]$and $\mathbf{L}=\left[\begin{array}{c}\ell_{+}^{T} \\ \ell_{-}^{T}\end{array}\right]$ is a matrix of left eigenvalues of $\mathbf{D F}$ such that $\mathbf{L} \cdot \mathbf{D F}=\boldsymbol{\Lambda} \cdot \mathbf{L}$. In $c_{1}=\sqrt{\frac{A}{\rho \mathcal{C}}}$ $0, L] \times[0, T]$, with uniform mesh-spacing $\Delta z$ and time step $\Delta t^{n}$. The cells are for hyperbolic problems:

Following Formaggia and Veneziani [19], system (10) is written in diago-

$$
\frac{1}{\Delta t^{n}}\left(U_{\mathfrak{i}}^{n+1}-U_{\mathfrak{i}}^{n}\right)+\frac{1}{\Delta z}\left(F_{\mathfrak{i}+1 / 2}^{n}-F_{\mathfrak{i}-1 / 2}^{n}\right)-S_{\mathfrak{i}}^{n}=0,
$$




$$
F_{\mathfrak{i}+\frac{1}{2}}=\frac{1}{2}\left[F\left(U_{\mathfrak{i}}^{n}\right)+F\left(U_{\mathfrak{i}+1}^{n}\right)-\lambda_{\mathfrak{i}+\frac{1}{2}}\left(U_{\mathfrak{i}}^{n+1}-U_{\mathfrak{i}}^{n}\right)\right]
$$

162 with $\lambda_{\mathfrak{i}+\frac{1}{2}}=\max \left\{\lambda_{-}\left(U_{\mathfrak{i}}^{n}\right), \lambda_{+}\left(U_{\mathfrak{i}+1}^{n}\right)\right\}$, and where $S_{\mathfrak{i}}^{n}=\left[\begin{array}{c}0 \\ -K_{r} \frac{Q_{i}^{n}}{A_{\mathfrak{i}}^{n}}\end{array}\right]$. Time-

where $\lambda=\lambda_{-0}^{n}$ and $\Delta t=\Delta t^{n}$. Similarly, we impose

$$
W_{1, \text { out }}^{n+1}=\frac{\Delta z-\lambda \Delta t}{\Delta z} W_{1 M+1}^{n}+\frac{\lambda \Delta t}{\Delta z} W_{1 M}^{n}
$$

174 where $\lambda=\left\{\lambda_{+}\right\}_{M}^{n}$. space $\Delta t^{n}$ must verify the well-known stability condition $2 \max _{\mathfrak{i}}\left|\lambda_{\mathfrak{i}+\frac{1}{2}}\right| \Delta t^{n} \leq \Delta z$.

Numerical boundary conditions are prescribed, thus $U_{i n}^{n}=U_{-1}^{n}$ and $U_{\text {out }}^{n}=$ $U_{M+1}^{n}$ are given to compute $U_{0}^{n+1}$ and $U_{M}^{n+1}$ at $z=0$ and $z=L$ respectively. Since flows involved in this model are sub-critical, the numerical scheme (14) requires one condition at each boundary. An admissible condition imposes the incoming characteristic and allows the wave corresponding to the outgoing characteristic to leave the domain. As $W_{2}$ remains constant along the characteristic curve defined by $\frac{d z}{d t}=\lambda_{-}(U(z, t))$, if we approximate $\lambda_{-}(U(z, t))$ by its numerical value $\lambda_{-}\left(U\left(z, t^{n}\right)\right)$ in $\left[t^{n}, t^{n+1}[\right.$, we consider that

$$
W_{2}\left(z, t^{n+1}\right) \simeq W_{2}\left(z-\left\{\lambda_{-}\right\}_{0}^{n} \Delta t^{n}, t^{n}\right),
$$

thus

$$
W_{2, i n}^{n+1}=\frac{\Delta z+\lambda \Delta t}{\Delta z} W_{2,-1}^{n}-\frac{\lambda \Delta t}{\Delta z} W_{2,0}^{n}
$$

\section{Boundary conditions}

We used MRI measured boundary conditions on the inlet of the tube phantom or the arterial segment. Flow measured on the entrance was imposed as a condition on the first mesh. We extrapolated, as explained above, the inlet value of $W_{2}$, and $A$ was computed by using relations (12) and (13). The outlet 
boundary condition is obtained by coupling the $1 \mathrm{D}$ model with a $0 \mathrm{D}$ model that consists of a system of differential equations linking pressure to flow at the 1D model exit. In fact, as shown in Alastruey et al. [4], the behavior happening beyond the 1D-modeled arteries can be represented by a 0D lumped-parameters model, also known as the Windkessel model, that simulates hemodynamics by electrical circuit analogy. Pressure gradient is represented by a potential difference, blood flow by the electrical current and hydraulic impedance by an electrical impedance. Hydraulic impedance combines friction loss, arterial wall elasticity and blood flow inertia, which are modeled by a resistance, a capacitor and an inductance respectively. A three-element (RCR) Windkessel model is a good compromise accounting for wall compliance and resistance in the peripheral network as well as proximal aortic impedance Wetterer [48], Westerhof et al. [47]. It is a combination of an R-model and an RC-model that improves considerably the behavior of the original two-element model proposed by Otto Frank Frank [20] by removing pressure and flow oscillations Stergiopulos et al. [43], Alastruey [1]. The first resistance $Z_{c}$ is the proximal aorta characteristic impedance, the compliance $C$ in parallel to the resistance $R_{2}$ simulate respectively the volume compliance and the resistance of the vascular network found downstream the abdominal aorta (Figure 2).

The inlet flow of the $\mathrm{R}$ model, which is the 1D-model outlet flow $\left(Q_{i n-0 D}=\right.$ $\left.Q_{\text {out }-1 D}\right)$, is:

$$
Q_{i n-0 D}=\frac{P_{i n-0 D}-P_{C}}{Z_{c}}
$$

As for the RC-model:

$$
C \frac{\mathrm{d} P_{C}}{\mathrm{~d} t}-\frac{P_{\text {out }-0 D}-P_{C}}{R_{2}}-Q_{i n-0 D}=0
$$


$P_{\text {out }-0 D}$ is the pressure at the $0 \mathrm{D}$-model exit. We compute $P_{C}$ at each time step by explicitly discretizing equation (16):

$$
P_{C}^{N+1}=f\left(P_{C}^{N}, Q_{i n-0 D}^{N}, P_{o u t-0 D}\right)
$$

then use the relation at the 1D-model exit:

$$
Q_{i n-0 D}^{N+1}=\frac{P_{i n-0 D}^{N+1}-P_{C}^{N+1}}{Z_{c}}
$$

\section{MRI experiments}

A straight flexible silicone tube was used as a phantom to validate the 1D-model. The MRI setup (Figure 3), described in a previous work Khalife et al. [23], consisted of the phantom connected to a non-magnetic CardioFlow 5000MR pump (Shelley Automation, Toronto, Canada) that can generate a programmable flow pattern. We used a blood mimicking fluid (BMF, $62 \%$ water and $38 \%$ glycerol) with similar magnetic and physical properties as blood. It has a Newtonian behavior which is a safe assumption for blood circulating in large arteries Ottesen et al. [33]. Two MRI-compatible optical pressure sensors (Opsens, QuÃl'bec, Canada) were introduced into the phantom. These sensors allow simultaneous pressure measurements used as validation for the simulation. To test the 1D-model application on a curved segment and estimate the error, the tube phantom was replaced with an aortic phantom (Elastrat, Geneva, Switzerland) comprising an ascending aorta branch (AAo) and a descending aorta branch (DAo). Pressure sensors were introduced at the AAo entrance and at the DAo exit.

A sinusoidal waveform of amplitude $150 \mathrm{~mL} \cdot \mathrm{s}^{-1}$ was applied in the tube phantom setup. In the aorta phantom setup, a flow wave similar to the one measured 
at the left ventricle exit was programmed on the pump control system with an amplitude of $250 \mathrm{~mL} \cdot \mathrm{s}^{-1}$ and a period of $0.8 \mathrm{~s}(75 \mathrm{bpm})$.

Experiments were performed on a 1.5T MRI system (Achieva; Philips, Best, the Netherlands) using SENSE Flex-L coils. Phase-Contrast (PC) flow-encoded gradient-echo multiphase sequences were performed to assess through-plane velocity on two slices, one situated at the phantom entrance with the first pressure sensor and the second at the phantom exit, with the second pressure transducer. Scan parameters: spatial resolution: $1 \times 1 \times 8 \mathrm{~mm}^{3}$, echo time/repetition time $\mathrm{TE} / \mathrm{TR}=5.75 / 9.59 \mathrm{~ms}$, velocity encoding $V_{\text {enc }}=80 \mathrm{~cm} \cdot \mathrm{s}^{-1}$ for a flowrate of $150 \mathrm{~mL} \cdot \mathrm{s}^{-1}$ and $V_{\text {enc }}=100 \mathrm{~cm} . \mathrm{s}^{-1}$ for a flowrate of $250 \mathrm{~mL} \cdot \mathrm{s}^{-1}, 40$ time frames per cardiac cycle with ECG-triggering on the pump signal.

For the in vivo feasibility of the 1D-model, 3 healthy subjects (male, ages 27 , 30 and 33) were included in our study after having filled out a written consent form. All measurements were performed on a 1.5T MRI system with SENSE cardiac coil. PC flow-encoded sequence covering the thoracic aorta was acquired during breath-hold and ECG-gated gradient echo sequence (spatial resolution: $1.1 \times 1.1 \times 8 \mathrm{~mm}^{3}, \mathrm{TE} / \mathrm{TR}=3.0 / 5.1 \mathrm{~ms}, V_{\text {enc }}=180 \mathrm{~cm} \cdot \mathrm{s}^{-1}, 40$ time frames per cardiac cycle). Data were acquired in an axial plane to measure through-plane velocity in the descending and the abdominal aorta (AbAo). A coronal plane of the aorta was acquired to measure the distance between velocity encoding sites.

Arm pressure was measured before and after MRI acquisitions and used as a reference for the 1D-model pressure simulations.

\section{Compliance measurements}

MRI images were processed using an in-house code. Aorta section was segmented semi-automatically and visually assessed by the operator on the magnitude image then flow waveforms were computed from velocity data provided by the corresponding phase images. The inlet flow is thus used as the model input 
condition.

MRI non-invasive measurements were used to determine phantoms and aortic compliance through PWV. The method, described in a previous work Khalife et al. [23], is based on the transit time (TT) method commonly used for the PWV estimationMurgo et al. [30], Avolio et al. [6] (Figure 4).

For each experiment, the linear compliance is determined from PWV based on the Bramwell-Hill theory: $\mathcal{C}=\frac{A}{\rho(P W V)^{2}}$ Bramwell and Hill [9], Vulliémoz et al. [46], where $A$ is the vessel lumen area and $\rho$ the fluid density.

\section{Simulations}

Simulations were performed on an in-house program using Matlab (the Mathworks, Natick, USA) by setting the domain geometry, fluid properties and MRI measured compliance for the pressure law. Flow $Q$, section area $A$ and pressure $P$ are computed on the whole domain as functions of time and space, then compared to measured data on the corresponding nodes.

The values of the input parameters were the following:

- Tube phantom : $L=40 \mathrm{~cm}, \mathcal{C}=0.0012 \mathrm{~cm}^{2} \cdot \mathrm{mmHg}^{-1}$. The RCR-model components were estimated from measurements: $Z_{c}=1.24 \cdot 10^{8}$ Pa.s.m ${ }^{-3}$ was computed using the approach proposed in Mitchell et al. [28], $R_{2}=$ $1.63 \cdot 10^{8}$ Pa.s.m ${ }^{-3}, C_{\text {per }}=0.288 \mathrm{~cm}^{3} \cdot \mathrm{mmHg}^{-1}$ and $P_{\text {out }-0 D}=0$.

- Aortic phantom: $L=21.5 \mathrm{~cm}, \mathcal{C}=0.051 \mathrm{~cm}^{2} \cdot \mathrm{mmHg}^{-1}, Z_{c}=2.63$. $10^{6}$ Pa.s.m ${ }^{-3}, R_{2}=1.07 \cdot 10^{8}$ Pa.s.m ${ }^{-3}, C_{p e r}=0.137 \mathrm{~cm}^{3} . \mathrm{mmHg}^{-1}$.

- In vivo : the RCR parameters at the outlet were taken from Alastruey et al. [4] because of the absence of invasive measurements. The characteristic impedance is $Z_{0}=\rho \frac{c_{0}}{A_{0}}$. The peripheral resistance is $R_{2}=R_{T}-Z_{c}$ where $R_{T}=1.89 \cdot 10^{8} \mathrm{~Pa} . \mathrm{s} . \mathrm{m}^{-3}$ is the complete vascular system resistance. 
The simulations were performed on a 100 meshgrid on a scope of 10 periods, with an average simulation time of $328 \mathrm{~s}$. $A$ and $Q$ are computed at each time step, $\Delta t=10^{-4} \mathrm{~s}$. To evaluate results, the root mean square error in \% (RMS) was calculated between computed and measured pressure waves.

Furthermore, to evaluate the equivalence between the pressure law (5) and the compliance pressure law (8) proposed in this work, two simulations were carried out with the same flow conditions, but with different pressure laws. The flow waveform and the model parameters used were those of a physiological scenario as proposed in Alastruey et al. [4]: $A_{0}=\pi \cdot 10^{-4} \mathrm{~m}^{2}, \beta_{0}=\frac{4}{3} \sqrt{\pi} h E$ for the $\beta_{0}$ pressure law, with $E=0.4 \cdot 10^{6} \mathrm{~Pa}, h=1.5 \cdot 10^{-3} \mathrm{~m}, C=7.85 \cdot 10^{-9} \mathrm{~m}^{3} / \mathrm{Pa}$, and compliance of $\frac{2 A_{0}^{3 / 2}}{\beta_{0}}$ for the compliance pressure law.

\section{Results}

The results of the equivalence test between the pressure law (5) and the compliance pressure law (8) are depicted in figure 5. Based on these curves, we can say that the two pressure laws provide nearly identical results which means that the $\beta_{0}$ pressure law agree well with the linear pressure law with $\mathcal{C}=\frac{2 A_{0}^{3 / 2}}{\beta_{0}}$, as in equation (9). The RMS between both pressure curves is $6.2 \%$. Hence, even if the pressure to section area relation is not perfectly linear in the range of physiological pressure, the error committed by considering it linear is negligible.

In phantom experiments, simulated pressure was compared to pressure sensor measurements.

The inlet flow and the simulated pressure within the tube phantom are shown in figure 6 for the sinusoidal flow waveform of amplitude $150 \mathrm{~mL} \cdot \mathrm{s}^{-1}$. Computed pressure curves show good agreement with the measured pressure in the tube phantom. RMS error computed between both is $8.15 \%$ and confirms the agreement of the model results with measurements.

Furthermore, the simulated pressure at the DAo outlet in the aortic phantom 
was compared to pressure sensor measurements acquired at the same location (Figure 7b). RMS between both pressure curves is $4.97 \%$. Velocity measurement noise caused oscillations on the MR measured flow which were reported on the simulated pressure. This is due to the relatively low velocity measured in the descending branch. Thus, the flow was filtered before simulations (Figure 7a).

Beyond this, pressure curves simulated by the 1D-model on a straight segment formed by the DAo and the AbAo in healthy volunteers are plotted in figure 8. Their contour is in good agreement with pressure curves reported in vivo in the literature Schnabel et al. [38], Murgo et al. [30]. Also, according to the reflection principle described in Salvi [37], the systolic pressure increases along the arterial tree and consequently the brachial systolic pressure overestimates the aortic systolic pressure up to $20 \mathrm{mmHg}$ Levick [25]. Thus in figure 8 , the simulated systolic pressure in the aorta is smaller than the systolic pressure measured on each volunteer. Mean arterial pressure (MAP) computed on the simulated and measured pressure is added in figure 8.

\section{Discussion}

In this work, we showed that by combining a $1 \mathrm{D}$ aorta model to non-invasive MRI velocity measurements, we were able to estimate aortic pressure. Flow MRI had commonly been used to derive pressure differences, so estimating a local pressure without having to set a null pressure point is a step forward in cardiovascular diagnosis.

The MR-measured flow was used as input for the 1D-model and a coupled 0D-model was used as an output condition. The pressure law, which relies on invasive measurements in formal studies, is replaced by a non-invasive pressure law based on the aortic compliance. This compliance was measured from MRI assessed PWV. The model simulations were successfully validated on an MRI setup with a straight phantom, an aortic arch phantom and in vivo. 
At first, the simulations were applied on a simple straight tube to respect the model conditions and simplifications. Pressure values obtained with simulations were in good agreement with measurements and showed satisfactory results as in previous studies Alastruey et al. [2]. Then, simulations were applied on an aortic phantom and results showed a negligible error when compared to pressure measurements, meaning that, in the physiological range of blood velocity, the 1D-model coupled with MRI flow measurements and subject-specific compliance pressure law would be enough to describe the aortic flow behavior. Results in vivo showed that it can estimate realistic pressure curves in the DAo, in agreement with the brachial pressure measured in the clinical routine. A bias was observed between the two brachial measurements carried out before and after the MRI exam due to the subject changing state going from agitated prior to the exam to rested state after the exam was completed. Another explanation could be the difference in the subjects position (seated vs. supine) during measurements. Consequently, the measured arm pressures might have been different than what the simulations would yield.

This 1D-model, which is frequently used to model the aorta, is based on strong assumptions, considering only radial and axisymmetric wall deformations, constant pressure on a section along the tube axis, thus only depending on time and on the axial coordinate $z$, dominance of axial velocity, absence of turbulence and neglecting the vessel wall inhomogeneities. Nevertheless, the model serves its initial role of estimating an absolute pressure in a simple and fast non-invasive manner, thus it is widely used in large artery modeling for its fair reproduction of the physiological configuration. Even if the model is one-dimensional and must be applied to a straight segment, its application to a curved aortic phantom did not greatly affect the agreement of simulated pressure and measured pressure at the phantom outlet. However, the effect of an 
362

363

364

inertance in the 0D-model represented by an inductor in the electrical circuit could be considered. The inertance element as proposed in Segers et al. [39] could absorb the oscillations which appear on the pressure curve.

The compliance based pressure law is simple, non-invasive and widely anchored in clinical practice and its association to the 1D-model may be more advantageous than the previously used pressure laws reported here. The model showed satisfactory results and good reproduction of invasive measurements recorded on a tube phantom. Additionally, we have shown that this simplified linear pressure law is in good agreement with the $\sqrt{A}$ law proposed in the literature.

Some improvements could be made to the 1D-model in the future. The model could be made more patient-specific by adapting the 0D-model parameters to the patient at the outlet boundary condition. Since these parameters are taken from the literature and are not patient-specific, previous work Willemet et al. [49] has proposed to measure peripheral resistance by introducing pressure catheters in the femoral artery. However, this is a disadvantage to a method which offers to be strictly non-invasive. Further studies are needed to propose a non-invasive way to measure the RCR parameters. Also, the model supposes a constant compliance along the artery which is a non-realistic condition when studying aortic disease or stenosis. This problem will be addressed in future studies.

In the future, it would be interesting to compare the pressure estimation found in our model with catheter measurements in an animal experiment. Also, more in vivo experiments would allow assessing the reliability and the repeatability of such estimation techniques in the aorta and to study pressure changes in patients suffering from cardiovascular diseases or under various stress conditions. 


\section{Conflict of interest statement}

The authors declare that there are no conflicts of interest.

\section{References}

[1] Alastruey, J., 2006. Numerical modelling of pulse wave propagation in the cardiovascular system: development, validation and clinical applications. Ph.D. thesis, Imperial College London.

[2] Alastruey, J., Khir, A. W., Matthys, K. S., Segers, P., Sherwin, S. J., Verdonck, P. R., Parker, K. H., Peiró, J., 2011. Pulse wave propagation in a model human arterial network: Assessment of 1-D visco-elastic simulations against in vitro measurements. Journal of Biomechanics 44 (12), 2250 -2258 .

URL http://www.sciencedirect.com/science/article/pii/S0021929011004386

[3] Alastruey, J., Nagel, S. R., Nier, B. A., Hunt, A. A., Weinberg, P. D., Peiró, J., 2009. Modelling pulse wave propagation in the rabbit systemic circulation to assess the effects of altered nitric oxide synthesis. Journal of Biomechanics 42 (13), 2116-2123.

URL http://www. sciencedirect.com/science/article/pii/S0021929009003182

[4] Alastruey, J., Parker, K. H., Peiró, J., Sherwin, S. J., AUG 2008. Lumped parameter outflow models for 1-D blood flow simulations: Effect on pulse waves and parameter estimation. Communications in Computational Physics 4 (2), 317-336.

[5] Alastruey, J., Passerini, T., Formaggia, L., Peiró, J., 2012. Physical determining factors of the arterial pulse waveform: theoretical analysis and calculation using the 1-D formulation. Journal of Engineering Mathematics 
77 (1), 19-37.

URL http://dx.doi.org/10.1007/s10665-012-9555-z

[6] Avolio, A., Chen, S.-G., Wang, R.-P., Zhang, C.-L., Li, M.-F., Orourke, M., 1983. Effects of Aging on Changing Arterial Compliance and LeftVentricular Load in a Northern Chinese Urban-Community. Circulation $68(1), 50-58$.

[7] Bessems, D., Giannopapa, C. G., Rutten, M. C., van de Vosse, F. N., 2008. Experimental validation of a time-domain-based wave propagation model of blood flow in viscoelastic vessels. Journal of Biomechanics 41 (2), $284-291$. URL http://www.sciencedirect.com/science/article/pii/S0021929007003922

[8] Bock, J., Frydrychowicz, A., Lorenz, R., Hirtler, D., Barker, A. J., Johnson, K. M., Arnold, R., Burkhardt, H., Hennig, J., Markl, M., OCT 2011. In vivo noninvasive $4 \mathrm{D}$ pressure difference mapping in the human aorta: phantom comparison and application in healthy volunteers and patients. Magnetic Resonance in Medicine 66 (4), 1079-1088.

[9] Bramwell, J., Hill, A., 1922. Velocity of transmission of the pulse-wave: and elasticity of arteries. The Lancet 199 (5149), $891-892$, originally published as Volume 1, Issue 5149.

URL http://www.sciencedirect.com/science/article/pii/S0140673600955806

[10] Buyens, F., Jolivet, O., De Cesare, A., Bittoun, J., Herment, A., Tasu, J., Mousseaux, E., APR 2005. Calculation of left ventricle relative pressure distribution in MRI using acceleration data. Magnetic Resonance in Medicine 53 (4), 877-884.

[11] Chen, C.-H., Nevo, E., Fetics, B., Pak, P. H., Yin, F. C., Maughan, W. L., Kass, D. A., 1997. Estimation of Central Aortic Pressure Waveform by 
Mathematical Transformation of Radial Tonometry Pressure: Validation of Generalized Transfer Function. Circulation 95 (7), 1827-1836.

URL http://circ. ahajournals.org/content/95/7/1827 . abstract

[12] Chen, C.-H., Ting, C.-T., Nussbacher, A., Nevo, E., Kass, D. A., Pak, P., Wang, S.-P., Chang, M.-S., Yin, F. C., 1996. Validation of Carotid Artery Tonometry as a Means of Estimating Augmentation Index of Ascending Aortic Pressure. Hypertension 27 (2), 168-175.

URL http://hyper . ahajournals.org/content/27/2/168. abstract

[13] Cohn, J., Quyyumi, A., Hollenberg, N., Jamerson, K., JUN 29 2004. Surrogate markers for cardiovascular disease functional markers. Circulation $109(25, \mathrm{~S}), 31-46$.

[14] Collaboration, P. S., 2002. Age-specific relevance of usual blood pressure to vascular mortality: a meta-analysis of individual data for one million adults in 61 prospective studies. The Lancet 360 (9349), 1903-1913. URL http://www.sciencedirect.com/science/article/pii/S0140673602119118

[15] Conrad, W., Oct 1969. Flow relationships in collapsible tubes. IEEE Transactions on Biomedical Engineering 16 (4), 284-295.

[16] Dumas, L., 2008. Inverse problems for blood flow simulation. In: EngOpt 2008-International Conference on Engineering Optimization, number June.

[17] Ebbers, T., Wigstro, L., Bolger, A. F., Engvall, J., Karlsson, M., 2001. Estimation of relative cardiovascular pressures using time-resolved threedimensional phase contrast MRI. Magnetic Resonance in Medicine 45, 872879.

[18] Formaggia, L., Lamponi, D., Quarteroni, A., 2003. One-dimensional models for blood flow in arteries. Journal of Engineering Mathematics 47, 251-276. 
[19] Formaggia, L., Veneziani, A., May 2003. Reduced and multiscale models for the human cardiovascular system. Lecture Series notes on Biological Fluid Dynamics at the Von Karman Institute, Belgium 7.

[20] Frank, O., 1899. Die grundform des arteriellen pulses. Z Biol 37 (483-526), 19.

[21] Hayashi, K., Handa, H., Nagasawa, S., Okumura, A., Moritake, K., 1980. Stiffness and elastic behavior of human intracranial and extracranial arteries. Journal of Biomechanics 13 (2), 175 - 184.

URL http://www.sciencedirect.com/science/article/pii/0021929080901918

[22] Hughes, T. J., Lubliner, J., 1973. On the one-dimensional theory of blood flow in the larger vessels. Mathematical Biosciences 18, $161-170$.

URL http://www.sciencedirect.com/science/article/pii/0025556473900278

[23] Khalife, M., Rodriguez, D., de Rochefort, L., Durand, E., 2012. In vitro validation of non-invasive aortic compliance measurements using MRI. Computer Methods in Biomechanics and Biomedical Engineering $15(\sup 1), 83-84$.

URL http: //www.tandfonline.com/doi/abs/10.1080/10255842.2012.713638

[24] Langewouters, G., Wesseling, K., Goedhard, W., 1984. The static elastic properties of 45 human thoracic and 20 abdominal aortas in vitro and the parameters of a new model. Journal of Biomechanics 17 (6), $425-435$.

URL http://www.sciencedirect.com/science/article/pii/0021929084900344

[25] Levick, J., 2003. An introduction to cardiovascular physiology. A Hodder Arnold Publication. Hodder Arnold.

[26] Liang, F., 2014. Numerical validation of a suprasystolic brachial cuff-based method for estimating aortic pressure. Bio-Medical Materials and Engi- 
neering 24, 1053-1062.

URL http://dx.doi.org/10.3233/BME-130903

[27] Martin, V., Clément, F., Decoene, A., Gerbeau, J.-F., 2005. Parameter identification for a one-dimensional blood flow model. ESAIM: Proc. 14, 174-200.

URL http://dx.doi.org/10.1051/proc: 2005014

[28] Mitchell, G., Tardif, J., Arnold, J., Marchiori, G., O'Brien, T., Dunlap, M., Pfeffer, M., DEC 2001. Pulsatile Hemodynamics in congestive heart failure. Hypertension 38 (6), 1433-1439, 4th International Workshop on Structure and Function on Large Arteries, PARIS, FRANCE, APR 27-28, 2001.

[29] Muhler, E., Neuerburg, J., Ruben, A., Grabitz, R., Gunther, R., Messmer, B., Vonbernuth, G., SEP 1993. Evaluation of Aortic Coarctation after Surgical Repair - Role of Magnetic-Resonance-Imaging and Doppler Ultrasound. British Heart Journal 70 (3), 285-290.

[30] Murgo, J. P., Westerhof, N., Giolma, J. P., Altobelli, S. A., 1980. Aortic input impedance in normal man: relationship to pressure wave forms. Circulation 62 (1), 105-16.

URL http://circ. ahajournals.org/content/62/1/105. short

[31] Olufsen, M., Peskin, C., Kim, W., Pedersen, E., Nadim, A., Larsen, J., NOV-DEC 2000. Numerical simulation and experimental validation of blood flow in arteries with structured-tree outflow conditions. Annals of Biomedical Engineering 28 (11), 1281-1299.

[32] O'rourke, M. F., Blazek, J. V., Morreels, C. L., Krovetz, L. J., 1968. Pressure Wave Transmission along The Human Aorta: >Changes with Age and in Arterial Degenerative Disease. Circulation Research 23 (4), 567-579. URL http://circres . ahajournals .org/content/23/4/567 . abstract 
[33] Ottesen, J. T., Olufsen, M. S., Larsen, J. K., 2004. Applied Mathematical Models in Human Physiology. Society for Industrial and Applied Mathematics.

[34] Park, M. K., Guntheroth, W. G., 1970. Direct Blood Pressure Measurements in Brachial and Femoral Arteries in Children. Circulation 41 (2), $231-237$.

URL http://circ. ahajournals.org/content/41/2/231. abstract

[35] Quarteroni, A., Formaggia, L., 2004. Mathematical modelling and numerical simulation of the cardiovascular system. In: Ayache, N. (Ed.), Computational Models for the Human Body. Vol. 12 of Handbook of Numerical Analysis. Elsevier, pp. 3 - 127.

URL http://www.sciencedirect.com/science/article/pii/S1570865903120017

[36] Reymond, P., Merenda, F., Perren, F., Rüfenacht, D., Stergiopulos, N., 2009. Validation of a one-dimensional model of the systemic arterial tree. American Journal of Physiology - Heart and Circulatory Physiology 297 (1), $\mathrm{H} 208-\mathrm{H} 222$.

URL http://ajpheart.physiology .org/content/297/1/H208

[37] Salvi, P., 2012. Pulse Waves: How Vascular Hemodynamics Affects Blood Pressure. Springer.

[38] Schnabel, T. G., Fitzpatrick, H. F., Peterson, L. H., Rashkind, W. J., Talley, D., Raphael, R. L., 1952. A technic of vascular catheterization with small plastic catheters: its utilization to measure the arterial pulse wave velocity in man. Circulation 5 (2), 257-262.

URL http://circ. ahajournals.org/content/5/2/257.abstract

[39] Segers, P., Rietzschel, E. R., De Buyzere, M. L., Stergiopulos, N., Westerhof, N., Van Bortel, L. M., Gillebert, T., Verdonck, P. R., 2008. Three- 
and four-element Windkessel models: Assessment of their fitting performance in a large cohort of healthy middle-aged individuals. Proceedings of the Institution of Mechanical Engineers, Part H: Journal of Engineering in Medicine 222 (4), 417-428.

URL http://pih.sagepub.com/content/222/4/417.abstract

[40] Skinner, J. S., Adams, P. C., 1996. Outpatient cardiac catheterisation. International Journal of Cardiology 53 (3), $209-219$.

URL http://www.sciencedirect.com/science/article/pii/0167527396025545

[41] Smith, N.P. Pullan, A., Hunter, P., 2000. Generation of an anatomically based geometric coronary model. Annals of Biomedical Engineering 28, 1425 .

URL http://dx.doi.org/10.1114/1.250

[42] Smith, N. P., 2004. A computational study of the interaction between coronary blood flow and myocardial mechanics. Physiological Measurement $25(4), 863$.

URL http://stacks.iop.org/0967-3334/25/i=4/a=007

[43] Stergiopulos, N., Westerhof, B. E., Westerhof, N., 1999. Total arterial inertance as the fourth element of the windkessel model. American Journal of Physiology - Heart and Circulatory Physiology 276 (1), H81-H88.

URL http://ajpheart.physiology.org/content/276/1/H81. abstract

[44] Thompson, R. B., McVeigh, E. R., 2003. Fast measurement of intracardiac pressure differences with 2D breath-hold phase-contrast MRI. Magnetic Resonance in Medicine 49, 1056-1066.

[45] Tyszka, M. J., Laidlaw, D. H., Asa, J. W., Silverman, J. M., 2000. Estimation of relative cardiovascular pressures using time-resolved three- 
dimensional phase contrast MRI. Journal of Magnetic Resonance Imaging $12,321-329$.

[46] Vulliémoz, S., Stergiopulos, N., Meuli, R., 2002. Estimation of local aortic elastic properties with MRI. Magnetic Resonance in Medicine 47 (4), 649654.

URL http://dx.doi.org/10.1002/mrm.10100

[47] Westerhof, N., Bosman, F., De Vries, C., Noordergraaf, A., 1969. Analog studies of the human systemic arterial tree. Journal of Applied Physiology $2(2), 121-143$.

[48] Wetterer, E., 1954. Flow and pressure in the arterial system, their hemodynamic relationship, and the principles of their measurement. Minnesota medicine 37 (2), 77 .

[49] Willemet, M., Lacroix, V., Marchandise, E., 2013. Validation of a 1D patient-specific model of the arterial hemodynamics in bypassed lowerlimbs: Simulations against in vivo measurements. Medical Engineering \& Physics 35 (11), 1573-1583.

URL http://www.sciencedirect.com/science/article/pii/S1350453313001057

[50] Yang, G.-Z., Kilner, P. J., Wood, N. B., Underwood, S. R., Firmin, D. N., 1996. Computation of flow pressure fields from magnetic resonance velocity mapping. Magnetic Resonance in Medicine 36, 520-526.

[51] Zananiri, F., Jackson, P., Halliwell, M., Harris, R., Hayward, J., Davies, E., Wells, P., DEC 1993. A Comparative-Study of Velocity-measurements in Major Blood-vessels using Magnetic-Resonance-Imaging and Doppler Ultrasound. British Journal Of Radiology 66 (792), 1128-1133. 


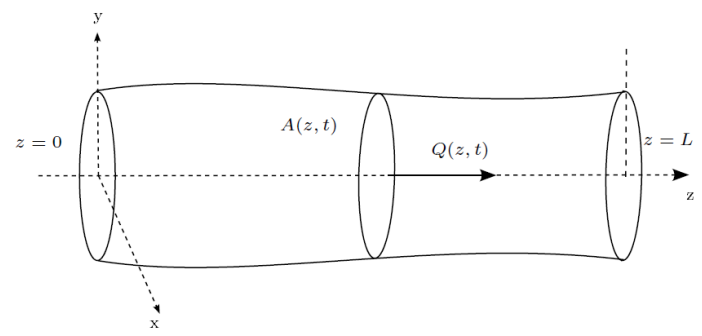

Figure 1: The 1D model simplified geometry. It assumes that the artery is a straight cylinder of length $L$ with a circular cross section $A(t, z)$ that deforms in respect to the radial vector.

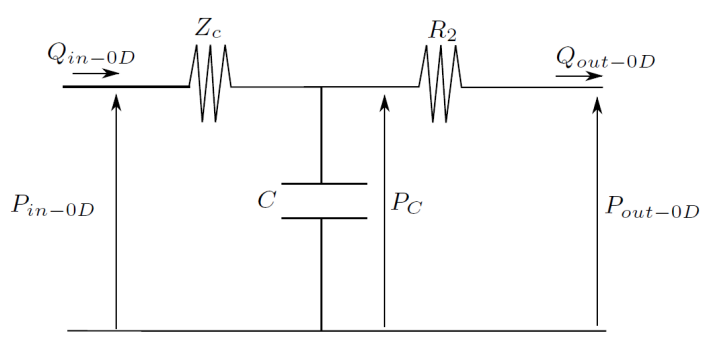

Figure 2: Coupling the 1D model outlet with the 0D model: a three-element Windkessel model (RCR) forms the outlet boudary condition of the 1D model. $\left(Q_{i n-O D}, P_{i n-O D}\right)=$ $\left(Q_{\text {out }-1 D}, P_{\text {out }-1 D}\right)$ and $\left(Q_{\text {out }-0 D}, P_{\text {out }-0 D}\right)$ are flow and pressure at the inlet and the outlet of the RCR model respectively. To solve the RCR model, two sub-models, RC and R, are considered and then combined. $P_{C}$ is the pressure across $C$ at the exit of the RC model and at the entrance of the $\mathrm{R}$ model.

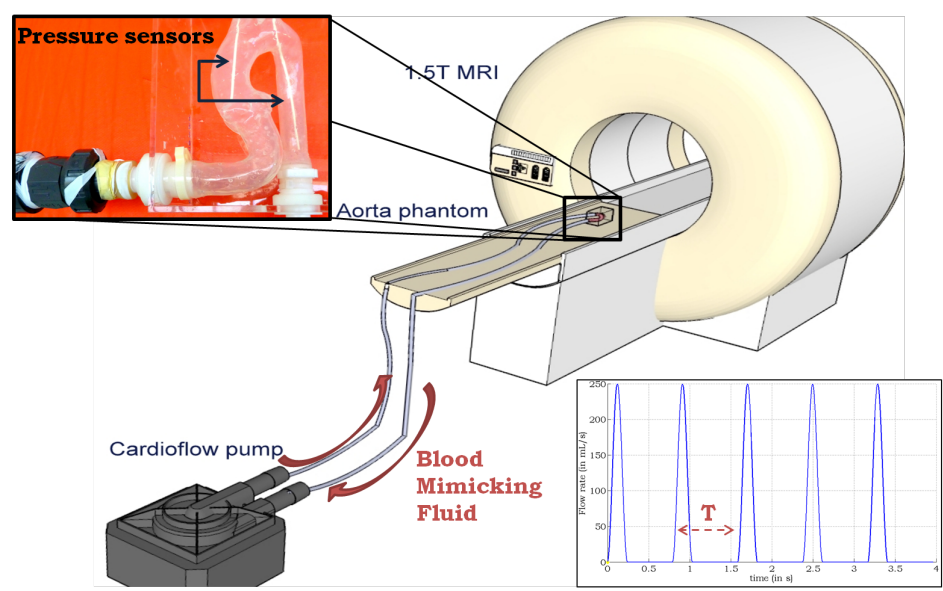

Figure 3: Experimental MRI setup with the MR-compatible pump and the aorta phantom. Optical pressure sensors are introduced in two locations on the phantom. 


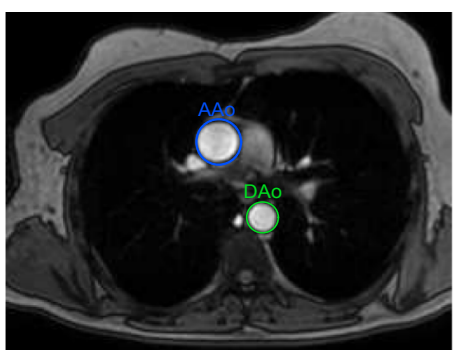

(a)

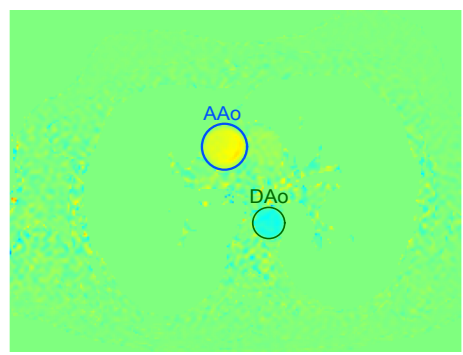

(b)

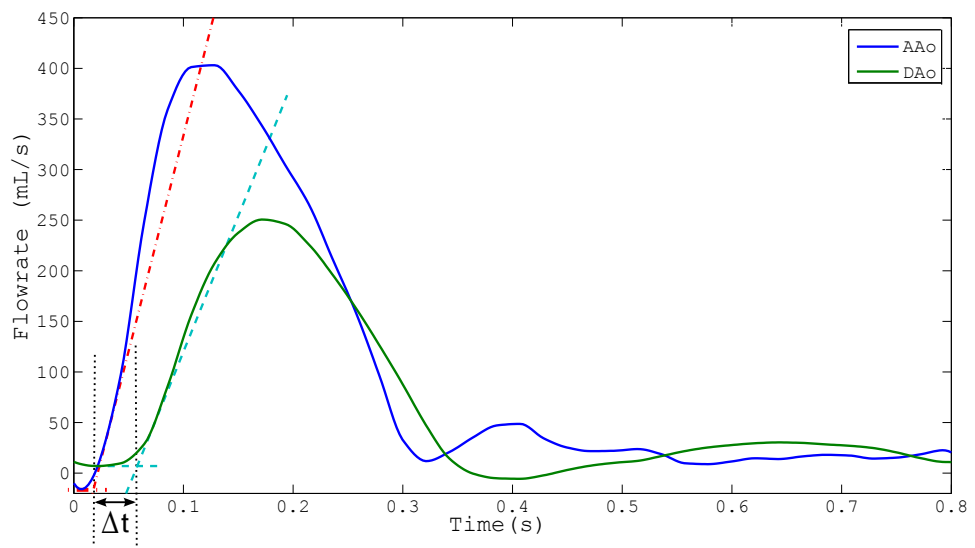

(c)

Figure 4: Transit time estimated on MR flow waves in subject A. (a) magnitude image of the acquired axial plane. (b) AAo and DAo are visible in hypersignal; through-plane velocity image in AAo and DAo : flow waveforms are extracted from the encoded velocity. (c) The TT method for PWV measurement. The TT, which is the delay $\Delta t$ that a waveform needs to travel a distance $L$, is used to compute the wave velocity: $P W V=\frac{L}{\Delta t}$. Here, $\Delta t$ was measured between the feet of flow waveforms extracted at two sections: tangent lines are obtained by the linear regression of the upslope segment on each waveform. 


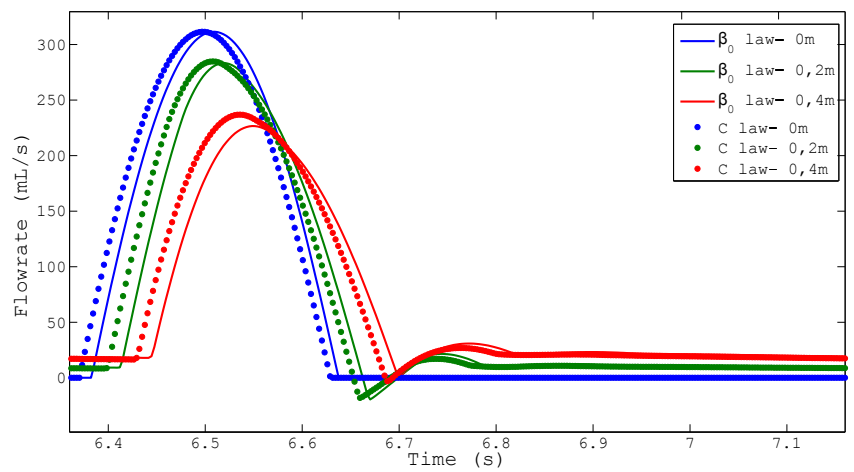

(a)

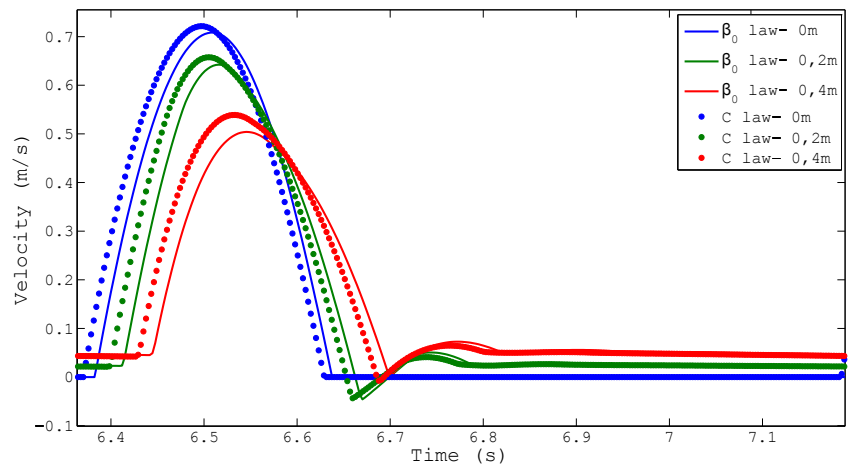

(b)

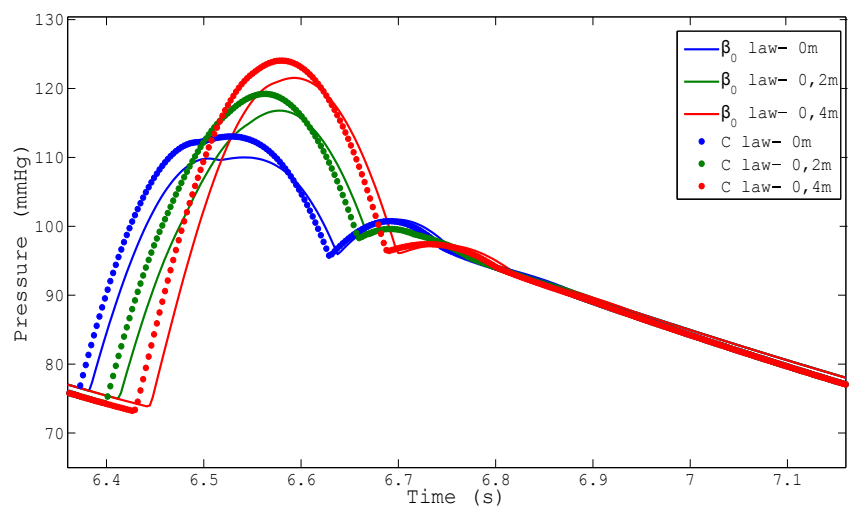

(c)

Figure 5: Results of the 1D model using pressure laws with $\beta_{0}$ in equation (5) (straight line) vs. with compliance $\mathcal{C}=\frac{2 A_{0}^{3 / 2}}{\beta_{0}}$ (dotted line). Simulations are performed with a pulsed ventricular flowrate and parameters given in Alastruey et al. [4]. Plotted results are such as: (a) flow waveform, (b) mean velocity and (c) pressure waveform simulated at three sites of the artery, at the inlet $(0 \mathrm{~m})$, in the middle $(0,2 \mathrm{~m})$ and at the exit $(0,4 \mathrm{~m})$. 


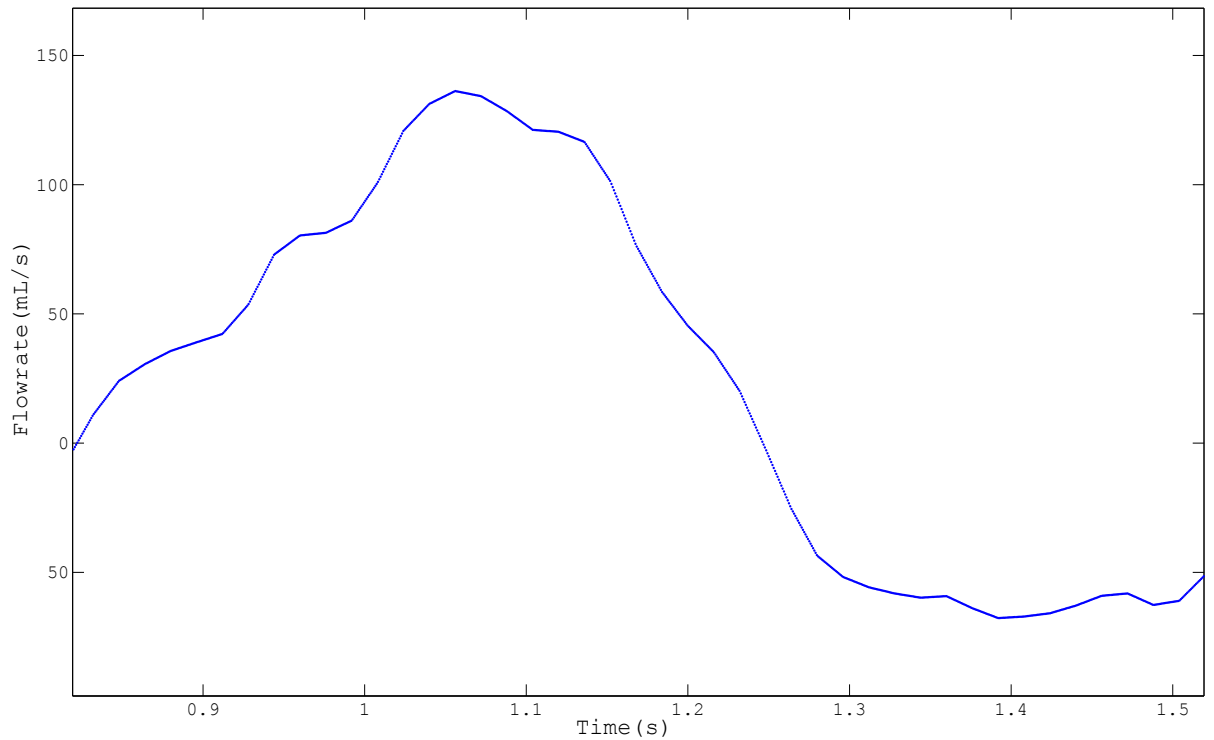

(a)

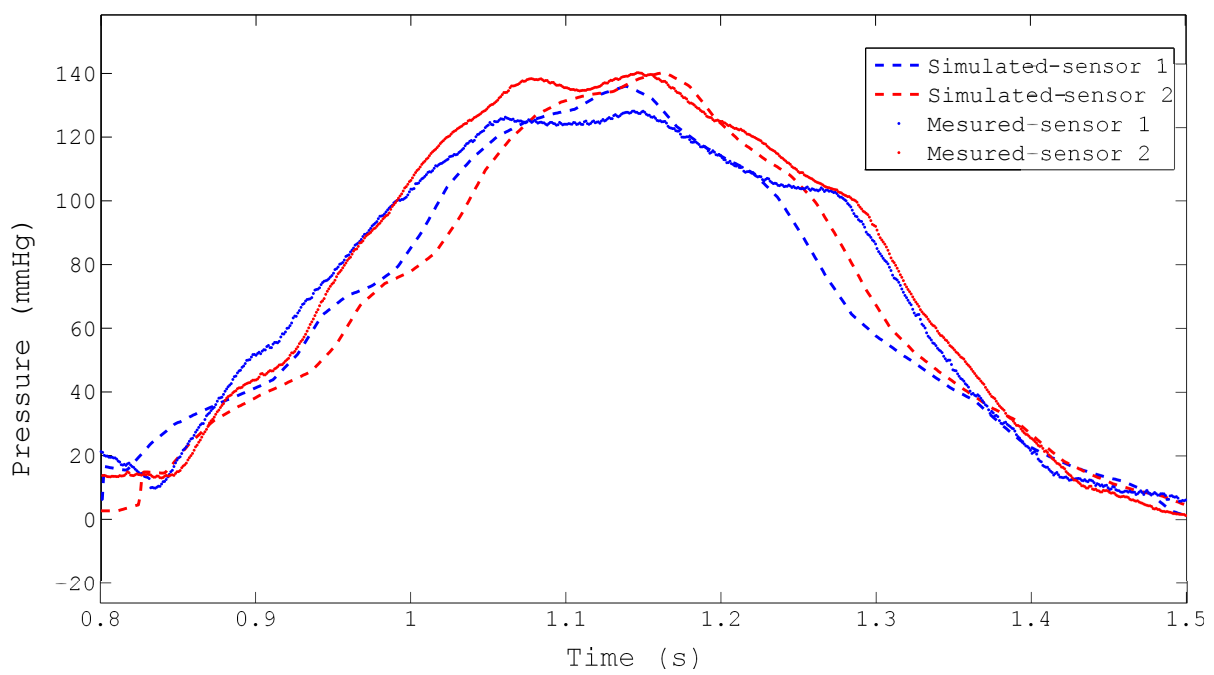

(b)

Figure 6: Pressure curves in the tube phantom with a sinusoidal flow waveform of amplitude $150 \mathrm{~mL} \cdot \mathrm{s}^{-1}$. Boundary conditions used are the measured flow at the inlet (a) and given by the 1D-0D coupled model at the outlet. (b) Simulated pressure curves at the sensors locations are compared to pressure measurements. 


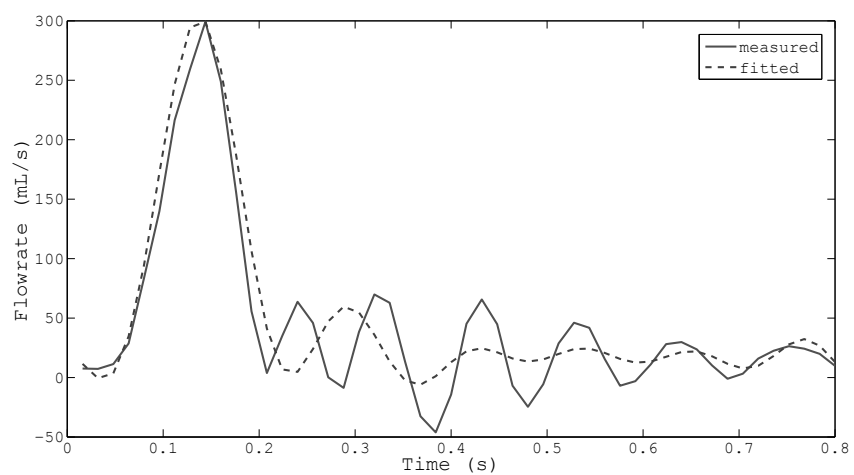

(a)

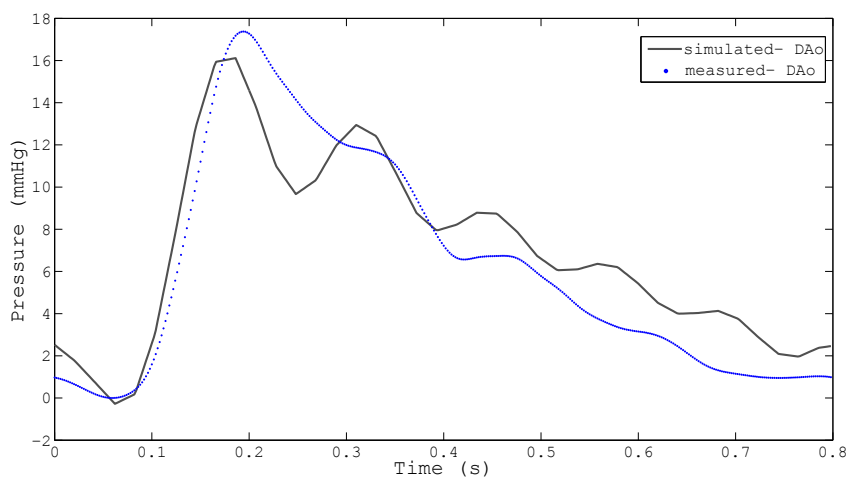

(b)

Figure 7: (a) Flow mesured at the entrance of the aorta phantom (solid line). Due to the motion of the phantom and the low velocity amplitude, oscillations are visible at the measurements curves and are reported in the 1D model simulation results. Hence, the fitted measured flow (dashed line) is used for pressure calculation. (b) Simulated vs. measured pressure at the exit of the aorta phantom with a ventricular flow of amplitude $250 \mathrm{~mL} . \mathrm{s}^{-1}$. Boundary conditions used are the flow at the entrance of the ascending aorta (AAo) and given by the $1 \mathrm{D}-0 \mathrm{D}$ coupled model at the outlet of the phantom. 


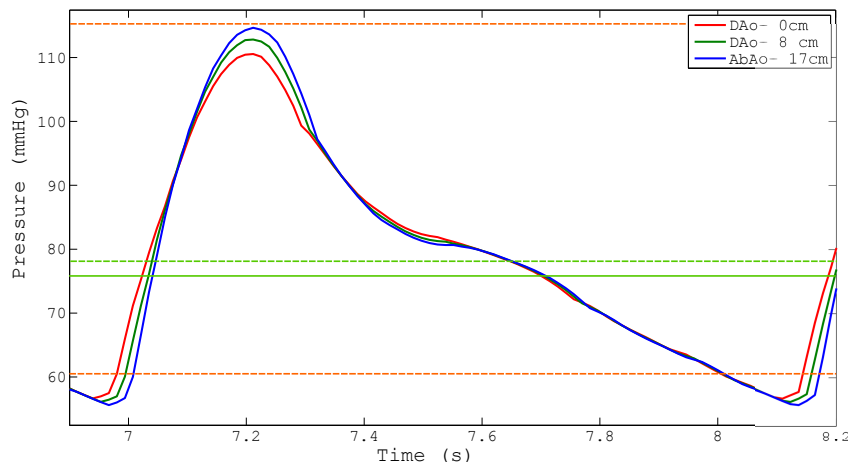

(a) subject A, 27 years old

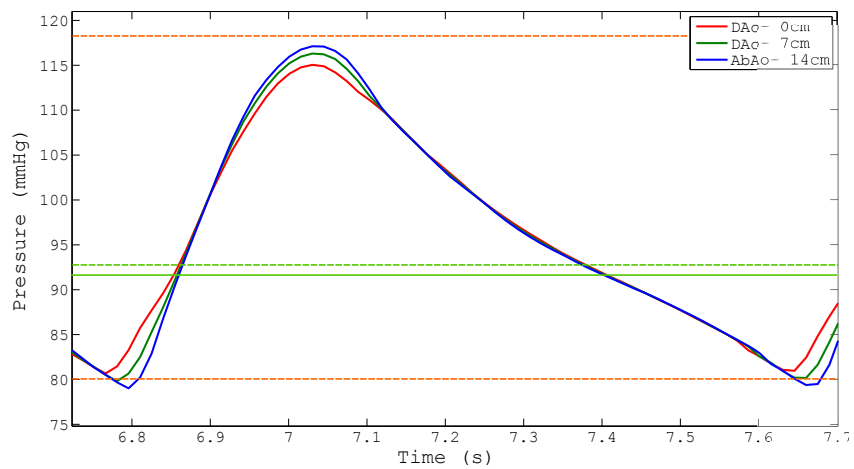

(b) subject B, 30 years old

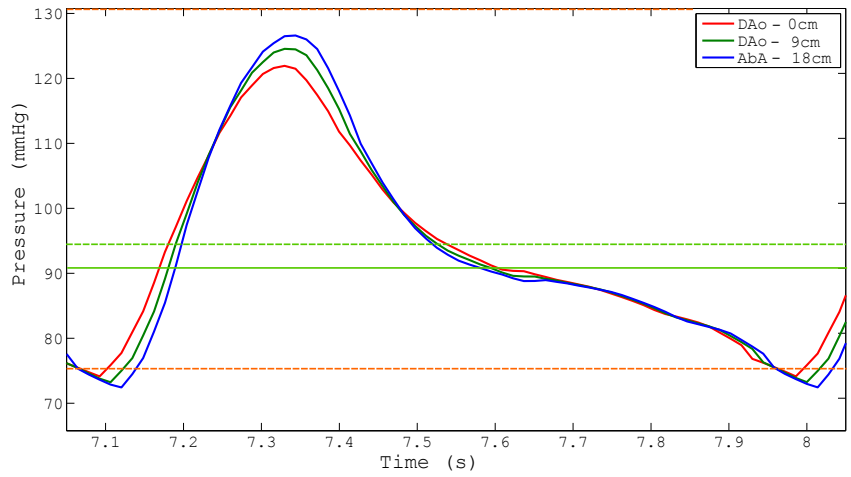

(c) subject C, 33 years old

Figure 8: Healthy volunteers' aortic pressure simulated in a straight segment going from the descending aorta (DAo) to the abdominal aorta $(\mathrm{AbAo})$. The horizontal dashed lines in orange show the averaged brachial diastolic and systolic pressure $\left(P_{\text {diast }}\right.$ and $\left.P_{\text {syst }}\right)$ measured for each subject. In green, the corresponding mean arterial pressure (MAP) lines are computed on the simulated pressure (solid line) and the arm measurements (dashed line). The inlet condition is the flow measured at the base of the descending aorta and the outlet condition is given by the 1D-0D coupled model. Pressure law based on compliance measurement (equation 8) is used. Mean arterial pressure is given by: $M A P=P_{\text {diast }}+\frac{1}{3}\left(P_{\text {syst }}-P_{\text {diast }}\right)$. 